P160 (continued)

www.ChooseMyPlate.gov. Students promoted the site through Facebook and posted on Instagram 3-4 times/ week. After six weeks, the 475 followers were sent an IRB approved survey.

Evaluation: Survey data were collected only from students at the university. Of the 72 respondents, almost all $(n=70)$ were female and most $(n=67)$ followed other nutrition related accounts. Most $(n=56)$ had heard of MyPlate prior to joining the account. Almost half $(n=32)$ reported using the nutrition tips somewhat frequently. Favorite features of the account were food pictures, healthy eating tips and recipes. Suggestions for improvement included more recipes and videos. All $(n=8)$ nutrition majors completed an IRB approved survey to assess their experience. Most $(n=7)$ felt their skills in providing nutrition education and leadership improved while many $(n=6)$ stated creative abilities increased. All indicated the project should continue and write-in responses showed students believe Instagram is a valuable way to show a healthy meal to peers.

Conclusions and Implications: Nutrition majors and non-majors benefitted from this project. Future programs could investigate whether Instagram is an effective educational tool for males and can further assess ways to promote healthy eating for females via pictures posted by peers.

Funding: None.

\section{P161 Conversations with High School Educators: Focus Groups Evaluating Diabetes Risk Factors and Resource Gaps in High Schools}

Nica Clark, MS, RDN, nica.clark@utah.edu, University of Utah, Department of Nutrition and Integrative

Physiology, 250 S 1850 E, Salt Lake City, UT 84112;

Julie Metos, PhD, MPH, RDN; Carmen Ramos, MS, RDN

Background (Background, Rationale, Prior Research, and/or Theory): U.S. adolescent obesity rates have increased from 6\% in 1971 to 20\% in 2011. Adolescents who are overweight or obese are more likely to develop type 2 diabetes. Few previous studies have addressed diabetes prevention among high school students. To our knowledge, no studies have utilized high school educators as key informants to identify factors influencing adolescent food and physical activity behaviors.

Objective: To identify the factors contributing to the increase in obesity and diabetes among adolescents, as perceived by high school educators.

Study Design, Setting, Participants, Intervention: High school health, physical education, and foods educators in a western state were invited for focus group participation. Educators responded to semi-structured interview questions in three categories: School Food and Physical Activity Environment, General Health Habits of High School Students, and Gaps in Nutrition and Physical Activity Resources.
Outcome Measures and Analysis: Complete transcripts of focus groups were thematically coded in NVivo 11 Pro for factors influencing adolescent diabetes risk and resource gaps.

Results: Four focus groups (two urban, two rural) were held, with 11 high school educators (eight females) participating. Teaching experience ranged from two to 20 years. Limited differences were observed in urban and rural schools. Thematic analysis revealed schools with declining emphasis on nutrition and physical activity. One semester health classes provide approximately six lessons on nutrition and physical activity. Recent state policy changes have decreased physical education requirements. Many parents and students lack knowledge about nutrition, exercise, and cooking; most lack application skills. Food insecurity is rampant, yet students and families chose convenience and fast foods regularly. Significant resource gaps exist. Programs utilizing peer modeling and technology may increase student engagement.

Conclusions and Implications: A successful diabetes prevention program should emphasize knowledge and application techniques for behavior change. Students may respond to programs based in technology and peer teaching. Cooking skills and family outreach may improve program success.

Funding: Larry H. and Gail Miller Family Foundation.

\section{P162 Developing a Food Production Record Template for Use as an Evaluation: Tool in School Lunchrooms}

Patrick J. Brady, MS, patrick-j-brady@uiowa.edu, University of Iowa College of Public Health, Public Policy Center, N465 College of Public Health Building, 145 N Riverside Drive, Iowa City, IA 52246;

Grace Ryan, MPH; Wensday Worth, BS;

Natoshia M. Askelson, PhD, MPH; Patti Delger, LD, RDN, Iowa Department of Education; Carrie Scheidel, MPH

Objective: The objective of this project was to develop an adaptable food production record template for the evaluation of nutrition interventions. This was accomplished through testing the multiple iterations of the template with food service staff (FSS) over two years.

Target Audience: Twenty-six FSS were selected to participate based on their involvement in two school lunch interventions offered by Iowa Team Nutrition.

Theory, Prior Research, Rationale: Production records provide information that can be used in the evaluation of lunchroom interventions. However, production record data are often incomplete or not detailed enough to be used for evaluation.

Description: Over the course of two years, a production record template was developed based on pilot testing of different forms, feedback from FSS and the USDA Iowa Advisory Council, and discussions among research staff. The final template collects the following information: quantity prepared, serving size, number of servings prepared, 